\title{
Avaliação da usabilidade do Guia Alimentar Digital móvel segundo a percepção dos usuários
}

\author{
Evaluation of the usability of a mobile Digital Food Guide \\ based on user perception
}

Simone Caivano ${ }^{1}$

Beatriz Jansen Ferreira ${ }^{2}$

Semíramis Martins Álvares Domene ${ }^{1}$

${ }^{1}$ Departamento de Políticas Públicas e Saúde Coletiva, Universidade Federal de São Paulo. R. Silva Jardim 136, Vila Mathias. 11.015-020 Santos SP Brasil. simone.caivano@hotmail.com ${ }^{2}$ Pró-Reitoria de Graduação, Universidade Estadual de Campinas.
Abstract The use of digital technology in the form of health care apps has been on the increase. In the nutrition area, apps are now available with a view to lead to behavior change, helping individuals to reflect on their food choices and identify weak points in their dietary routine. The article seeks to evaluate user perception regarding the usability of the Digital Food Guide (DFG), whi$c h$ is a mobile smartphone app with guidelines on healthy eating. A cross-sectional study evaluated the user perception of the app using the Likert scale, built with 24 assertions organized in three dimensions of analysis: the DFG as an intuitive and self-explanatory tool; the DFG as a promoter of healthy food choices; and the DFG as a promoter of the transition to the appropriate weight. The instrument was assessed regarding its reliability through the split-half and validity method in two stages. The 22 assertions were validated; the reliability was 0.93; the average of the assertions in each dimension was 3.10; of the 80 respondents, $58.75 \%$ considered the implementation of the DFG to be positive. The application has good usability as perceived by users, considering analysis of the dimensions relating to its performance. Key words Food education, Biomedical techno$\log y$, Instrument of perception, User satisfaction
Resumo O uso da tecnologia digital na forma de aplicativos para cuidados em saúde tem crescido; na área de nutrição estão disponíveis com o propósito de levar à mudança de comportamento, auxiliando individuos a refletirem sobre escolhas alimentares e identificarem fragilidades na rotina alimentar. $O$ artigo tem por objetivo avaliar a percepção dos usuários em relação à usabilidade do Guia Alimentar Digital (GAD), aplicativo para celular smartphone, com diretrizes sobre alimentação saudável. Estudo transversal avaliou a percepção de usuários do aplicativo por meio de escala Likert, construída com 24 asserções organizadas em três dimensões de análise: 1. GAD como ferramenta intuitiva e autoexplicativa; 2. GAD como promotor de escolhas alimentares saudáveis; 3. GAD como promotor da transição para o peso adequado. O instrumento foi analisado quanto à confiabilidade pelo método split-half e validade em duas etapas. Foram validadas 22 asserções; a confiabilidade foi de 0,93; a média de pontos das asserções em cada dimensão foi 3,10; dos 80 respondentes, $58,75 \%$ considerou positiva a implementação do GAD. O aplicativo apresenta boa usabilidade, segundo a percepção dos usuários, a considerar as análises das dimensões referentes ao seu desempenho.

Palavras-chave Educação alimentar, Tecnologia biomédica, Instrumento de percepção, Satisfação do usuário 


\section{Introdução}

Dados da Organização Mundial da Saúde permitem estimar que mais de um bilhão e quinhentos milhões de adultos estão acima do peso e que quinhentos milhões destes são clinicamente obe$\operatorname{sos}^{1}$. No Brasil, segundo a Pesquisa de Orçamentos Familiares realizada em 2008 e 2009, a população adulta apresentava 50,1\% de indivíduos com excesso de peso e $12,4 \%$ com obesidade ${ }^{2}$. A mudança de hábitos alimentares é reconhecida como estratégia de valor para a abordagem deste qua$\mathrm{dro}^{3,4}$ e o acompanhamento da ingestão alimentar é um componente que pode ajudar a monitorar o progresso em direção a escolhas saudáveis ${ }^{3}$.

$\mathrm{O}$ uso da tecnologia digital na forma de aplicativos para cuidados em saúde constitui um recurso com potencial para facilitar o registro alimentar e a análise dos dados em estudos dietéti$\cos ^{5,6}$; como atributo adicional, o uso desta tecnologia torna a coleta dos dados mais precisa e menos árdua, como se observou por meio de diversos tipos de ferramentas para a medição da ingestão alimentar ${ }^{5-8}$.

É crescente o acesso da população à tecnologia: segundo o Instituto Brasileiro de Geografia e Estatística (IBGE) $40,65 \%$ da população tinha acesso à internet no ano de $2010^{\circ}$. Em 2008, mais da metade $(53,8 \%)$ da população tinha telefone celular para uso pessoal - percentual que era de $36,6 \%$ em $2005^{10}$. Entre 2003 e 2006, os serviços de internet cresceram de 1,3 para $2,3 \%$ e a telefonia móvel cresceu de 34,1 para $43,2 \%$ no país ${ }^{11}$. Estes números demonstram o interesse da população brasileira pela tecnologia digital. Atualmente, mais de 1,3 bilhão de aparelhos celulares estão sendo vendidos no mundo; e em 2010 os smartphones compuseram cerca de $20 \%$ do total e as vendas crescem $100 \%$ ao ano ${ }^{12}$. Entende-se, portanto, que estas tecnologias podem influir positivamente sobre os hábitos dos indivíduos, como aqueles relacionados ao trabalho, ao ensino e à aprendizagem ${ }^{13}$.

As características dos aparelhos móveis permitem acesso à informação a qualquer hora (justin-time), o que pode ser a base para a individualização e personalização da aprendizagem; permitem distribuir, agregar e partilhar informação facilmente. O conceito de apropriação destes aparelhos é definido como a integração de uma nova ferramenta às atividades de aprendizagem ${ }^{13}$.

Já estão disponíveis em diversos países ferramentas digitais para o monitoramento alimen- tar e que auxiliam os indivíduos a ampliar sua consciência sobre escolhas apropriadas ${ }^{3}$; contudo, pouco se conhece sobre a percepção dos usuários sobre a usabilidade destes aplicativos e seu efeito sobre eventual mudança de comportamento. Em estudos internacionais sobre utilização de tecnologias, o gênero tem se destacado e é uma variável importante, já sendo comprovadas diferenças de comportamento entre homens e mulheres na utilização da tecnologia para ensino (elearning). Já no Brasil, os estudos sobre esse tema ainda são pouco explorados ${ }^{14}$, bem como a especificação por faixa etária.

Entre os protocolos disponíveis para medir percepção e comportamento está a escala de percepção atitudinal proposta por Likert ${ }^{15,16}$, que indica o grau de concordância ou discordância em reação à atitude que está sendo medida.

O Guia Alimentar Digital (GAD), objeto deste estudo, é um produto de inovação tecnológica, lançado em abril de 2009 por empresa de consultoria em alimentação e nutrição, a partir de projeto de parceria com a universidade. Disponível atualmente para as plataformas iOS e Android, pode ser conhecido por meio do link http:/ /www.nutraeviva.com.br/Site/programas-nutrabem/nutrabem-mobile. O GAD foi concebido a partir de consulta aos Guias Alimentares disponíveis e de diretrizes sobre alimentação saudável resultantes de consensos internacionais, sobretudo aqueles propostos pela Escola de Saúde Pública da Universidade de Harvard, liderada pelo pesquisador Walter C. Willett ${ }^{17,18}$, com ajustes para a valorização de alimentos com importância para o padrão alimentar brasileiro, como as leguminosas e da oferta de nutrientes sem uso de suplementos; optou-se ainda por não incluir fontes de álcool. Desde o lançamento do GAD até maio de 2012, quando se iniciou este estudo, 23.137 indivíduos adquiriram a ferramenta. A partir de estudo piloto foi possível observar uma tendência para uma boa aceitação da utilização desta ferramenta.

Este estudo tem o objetivo de avaliar a percepção dos usuários, bem como a diferença entre os sexos e a faixa etária, frente à usabilidade de um aplicativo para celular smartphone com diretrizes sobre alimentação saudável, no qual o GAD está disponível, identificando os pontos facilitadores e os obstáculos para sua utilização; apresenta como hipótese uma boa percepção dos usuários em relação à usabilidade desta ferramenta. 


\section{Métodos}

Construção do instrumento de percepção. A escala atitudinal Likert tem como característica a construção simples; permite a introdução de qualquer asserção que seja coerente ao resultado final, mesmo que não tão explícita à atitude estudada; a abrangência da resposta possibilita uma informação mais precisa de cada asserção ${ }^{19}$. A partir dos aspectos de interesse, foram criadas as dimensões de análise, elaboradas e distribuídas as asserções nestas dimensões, seguindo os critérios de uma escala atitudinal Likert, dentre eles: número máximo de 70 asserções, preenchimento em até 20 minutos, asserções distribuídas pelo pesquisador de acordo com a análise que se deseja realizar e intercalando asserções positivas e negativas $^{20}$; asserções semelhantes dentro da mesma dimensão de análise têm como objetivo assegurar a legitimidade e a consistência da resposta ${ }^{15,16}$. As dimensões e as asserções deste instrumento estão distribuídas da seguinte maneira:

- Dimensão I: GAD como uma ferramenta intuitiva e autoexplicativa. Asserções: 3, 6, 9, 12, $15,18,20,22,24$;

. Dimensão II: GAD como promotor de escolhas alimentares saudáveis. Asserções: 2, 5, 8, 11, 14, 17, 19, 23;

. Dimensão III: GAD como promotor da transição para o peso adequado. Asserções: 1, 4, 7, 10, 13, 16, 21.

Estudo de confiabilidade. Para medir a confiabilidade do instrumento aplicou-se o método split-half ${ }^{1}$. Computou-se para cada respondente, a soma dos pontos das asserções ímpares e, separadamente, a soma dos pontos das asserções pares, de forma a simular a situação de duas aplicações do instrumento; na sequência, procedeu-se o cálculo do coeficiente da correlação linear entre os valores mencionados. O coeficiente de confiabilidade do instrumento foi calculado utilizando-se a fórmula de Spearman-Brown ${ }^{21}$ com o objetivo de conhecer se o grupo responderia da mesma forma ao instrumento em outra oportunidade, adotando-se como critério de aceitação um mínimo de $80 \%$ de satisfação quanto a sua confiabilidade $(R \geq 0,80)$.

Estudo de validação. A validação de conteúdo das asserções foi verificada por meio de estudo de correlação linear (r), com o objetivo de assegurar a dispersão mínima de respostas entre os respondentes em relação à escala atitudinal proposta e à existência de consistência entre pontuação baixa na asserção e pontuação total baixa no instrumento, e vice-versa. Para o processo de validação estatística de escalas atitudinais como a de Likert, cabem medidas como o coeficiente de correlação linear, os testes Alpha de Cronbach e Chi-quadrado de Pearson. Neste estudo optou-se pela correlação linear. O valor de (r) foi calculado para todas as asserções na simulação da primeira administração, visando à sua depuração com a eliminação das asserções com correlação linear inferior a 0,30 . Na sequência, o valor de (r) foi calculado novamente no que se denomina segunda administração, levando-se em consideração apenas as asserções validadas na primeira, envolvendo todos os instrumentos respondidos. Mais uma vez, foram eliminadas asserções, agora com correlação linear inferior a 0,20 .

Estudo da percepção dos usuários do aplicativo. Seguindo os preceitos para aplicação da escala atitudinal Likert, foram atribuídos valores numéricos às respostas de cada asserção para refletir a força e a direção da reação do indivíduo: a concordância recebe valor alto enquanto a discordância recebe valor baixo; a pontuação total de cada atitude é resultante da média das pontuações obtidas ${ }^{15}$. As asserções validadas foram associadas à escala atitudinal de concordância plena à discordância plena, com termos intermediários, inclinado a concordar e inclinado a discordar ${ }^{18}$; esta escala teve uma associação numérica de intervalo constante, no caso 4, 3, 2, 1 ou $1,2,3,4$, a depender do fato da asserção ser favorável ou desfavorável ${ }^{20}$. Optou-se pelo uso de quatro opções de resposta para evitar tendência centrante, que traria viés para a análise dos resultados ${ }^{15}$. A partir desta pontuação é calculada a média de cada dimensão, o que revela a "qualidade" da percepção sobre o objeto pesquisa$\mathrm{do}^{19}$. Assim, é possível adotar a seguinte interpretação:

. de 4 a 3 pontos - percepção positiva do objetivo avaliado;

. de 2 a 2,99 pontos-percepção que indica que existem acertos no objeto avaliado, mas é desejável a implantação de melhorias;

. de 1 a 1,99 ponto - percepção ruim sobre o objeto avaliado, o que implica em mudanças ou substituições.

Calculou-se a prevalência entre as faixas de interpretação e aplicou-se o Chi-quadrado com intervalo de confiança de 0,05; para explorar os resultados em cada uma das dimensões também foram calculadas as médias, conforme diretrizes utilizadas em escalas atitudinais Likert; na comparação de médias entre os sexos e faixa etária foi utilizado o teste Mann-Whitney. Como a Dimensão III avalia a percepção dos usuários com 
o GAD sendo promotor da transição para o peso adequado, para análises que envolvem esta dimensão foram considerados somente os usuários que tinham o desejo de perder peso.

É fundamental esclarecer que esta percepção será um retrato conceitual ou prático, em função daquilo que foi afirmado ou negado nas asserções que compõem cada dimensão.

Estudo da usabilidade. Realizou-se estudo transversal a partir do envio aos usuários do aplicativo, por e-mail, de link para acesso ao instrumento de percepção abrigado na ferramenta Question Pro.

Sujeitos da pesquisa. Amostra de conveniência foi constituída com os indivíduos que buscaram voluntariamente o uso do aplicativo em que o GAD está disponível. Foram incluídos no estudo indivíduos de ambos os sexos, com idade entre 19 e 50 anos e que aceitaram participar da pesquisa por meio de concordância com o Termo de Consentimento Livre e Esclarecido (TCLE). Somente permaneceram no estudo aqueles indivíduos que utilizaram o aplicativo por pelo menos 15 dias, que possuíam pelo menos três refeições registradas e que preencheram o instrumento de percepção de forma completa.

Aspectos Éticos. Este estudo foi aprovado pelo Comitê de Ética em Pesquisa da Universidade Federal de São Paulo.

Para contato com os usuários foi feita uma atualização do aplicativo, com a inclusão do TCLE na tela de abertura, que permaneceu disponível por um período de seis meses (novembro de 2011 a maio de 2012).

\section{Resultados}

Dos 662 usuários que responderam afirmativamente por meio da concordância com o TCLE, 80 (34 homens e 46 mulheres) atenderam aos critérios de inclusão; somente dois indivíduos não tinham o desejo de perder peso, portanto para as análises que envolvem a Dimensão III, foram considerados os resultados de 78 indivíduos.

Confiabilidade e validade das asserções do instrumento. O estudo sobre a confiabilidade do instrumento resultou em um coeficiente igual a 0,93. A Tabela 1 contém os resultados da análise de asserção da primeira e da segunda administração do instrumento. O número de asserções validadas foi 22. Na primeira administração, a média geral foi de 3,04 pontos. As correlações lineares entre pontuação em cada asserção e de pontos do instrumento todo foram baixas (cor- relação $1<0,30)$ nas seguintes asserções: Utilizo o aplicativo só para contar calorias (1); A definição do perfil de atividade física do aplicativo é clara (3) sendo, esta última, negativa; portanto essas asserções foram desconsideradas. Na segunda etapa, a média geral do instrumento foi de 3,05 pontos, levando-se em conta somente as asserções validadas.

Percepção dos usuários quanto à usabilidade do aplicativo. A percepção foi positiva para $58,75 \%$ dos usuários e $41,25 \%$ indicaram que o aplicativo deve ter melhorias; nenhum dos usuários demonstra uma percepção negativa $(\mathrm{p}=$ $0,165)$. Conforme se depreende das notas médias por dimensão (Tabela 2), houve percepção positiva em todos os casos.

A Figura 1 trata dos resultados por dimensão segundo o sexo dos respondentes; na Figura 2 estão os resultados das três dimensões segundo a faixa etária de usuários de ambos os sexos.

\section{Discussão}

A forma de funcionamento do GAD se constitui uma novidade para a população brasileira, tendo em vista a escassez de produtos desta natureza; é também parca a literatura a respeito; este trabalho é pioneiro ao descrever a percepção de usuários sobre o uso da tecnologia digital para informação e autocuidado na área da saúde. O Brasil ocupa o $72^{\circ}$ lugar entre os 156 países avaliados sobre o Índice Integrado de Telefonia, Internet e Celular de Inclusão Digital, realizado pela Fundação Getúlio Vargas com base no Censo 2010 do IBGE 22.

Para a análise de confiabilidade, o coeficiente foi de 0,93 , o que reflete a boa aceitação do aplicativo. No uso da escala atitudinal Likert, considera-se adequada a perda de até 30 a $40 \%$ do total de assertivas, o que corresponderia de 7 a 10 itens ${ }^{16}$. A perda deste estudo foi de apenas duas assertivas $(8,34 \%)$; isto denota a qualidade da validação de conteúdo, considerando aspectos como adequação da fraseologia, objetividade e assertividade nas proposições apresentadas.

O GAD, objeto deste estudo, foi concebido com o propósito de apresentar três características para contribuir para o autocuidado: a possibilidade de uso fácil, a capacidade de promoção de escolhas alimentares saudáveis e a manutenção do peso corporal adequado. Assim, médias, medianas e frequência das três dimensões de análise dentro do intervalo de classificação denominado "percepção positiva", indicam uma boa per- 
Tabela 1. Resultado da análise de asserções do Guia Alimentar Digital; nota média por asserção e resultado da correlação linear. Brasil, 2012.

\begin{tabular}{lcccc}
\hline \multirow{2}{*}{ Asserções } & \multicolumn{2}{c}{ Primeira Administração } & & \multicolumn{2}{c}{ Segunda Administração } \\
\cline { 2 - 3 } \cline { 5 - 5 } & Média & Correlação 1 & Média & Correlação 2 \\
\hline 1 & 2,15 & 0,20 & & \\
2 & 3,19 & 0,68 & 3,19 & 0,67 \\
3 & 2,61 & $-0,41$ & & 0,67 \\
4 & 3,39 & 0,64 & 3,39 & 0,67 \\
5 & 3,08 & 0,66 & 3,08 & 0,49 \\
6 & 3,51 & 0,47 & 3,51 & 0,58 \\
7 & 2,99 & 0,56 & 2,99 & 0,48 \\
8 & 2,84 & 0,48 & 2,84 & 0,42 \\
9 & 3,59 & 0,40 & 3,59 & 0,51 \\
10 & 3,26 & 0,51 & 3,26 & 0,59 \\
11 & 3,21 & 0,57 & 3,21 & 0,40 \\
12 & 2,86 & 0,39 & 2,86 & 0,45 \\
13 & 2,73 & 0,45 & 2,73 & 0,72 \\
14 & 3,24 & 0,73 & 3,24 & 0,50 \\
15 & 2,79 & 0,50 & 2,79 & 0,70 \\
16 & 2,96 & 0,72 & 2,96 & 0,68 \\
17 & 2,83 & 0,68 & 2,83 & 0,51 \\
18 & 2,7 & 0,52 & 2,7 & 0,58 \\
19 & 3,41 & 0,57 & 3,41 & 0,63 \\
20 & 3,14 & 0,60 & 3,14 & 0,73 \\
21 & 3,26 & 0,72 & 3,26 & 0,64 \\
22 & 2,88 & 0,62 & 2,88 & 0,63 \\
23 & 2,99 & 0,66 & 3,35 & \\
24 & 3,35 & 0,62 & & \\
\hline
\end{tabular}

Tabela 2. Média, mediana e desvio padrão de acordo com a pontuação nas respostas dos usuários do Guia Alimentar Digital quanto às três dimensões: usabilidade, promoção de escolhas alimentares saudáveis e perda de peso. Brasil, 2012.

\begin{tabular}{lccc}
\hline \multicolumn{1}{c}{ Dimensão } & Mediana $^{*}$ & Média $^{*}$ & Desvio padrão $^{*}$ \\
\hline I - usabilidade & 3,31 & 3,10 & 0,44 \\
II - promoção de escolhas alimentares saudáveis & 3,13 & 3,10 & 0,52 \\
III - promoção para perda de peso & 3,17 & 3,11 & 0,51 \\
Total & 3,06 & 3,10 & 0,45 \\
\hline
\end{tabular}

"Máximo de 4 pontos.

cepção dos usuários quanto ao potencial do aplicativo; assim como uma construção tecnológica assertiva. Em estudo com 31 participantes, Boushey et al. ${ }^{23}$ aplicaram escala de satisfação com sete pontos e observaram que o uso de PDA (Personal Digital Assistants) foi preferido entre adolescentes para o registro alimentar comparativamente a responder um recordatório de 24 horas aplicado com uma repetição; a percepção favorável sobre o uso da tecnologia digital também foi positiva em estudo com 129 usuários diabéti$\cos ^{24}$; os resultados de aplicação da escala Likert, também adotada pelos autores, mostraram que 87,9\% dos usuários reconhecem a utilidade do PDA para automonitoramento.

Ao analisar os resultados segundo o sexo dos respondentes (Figura 1), observa-se que as percepções foram muito semelhantes entre homens e mulheres quanto à usabilidade do aplicativo (Dimensão I) e o GAD como promotor da tran- 


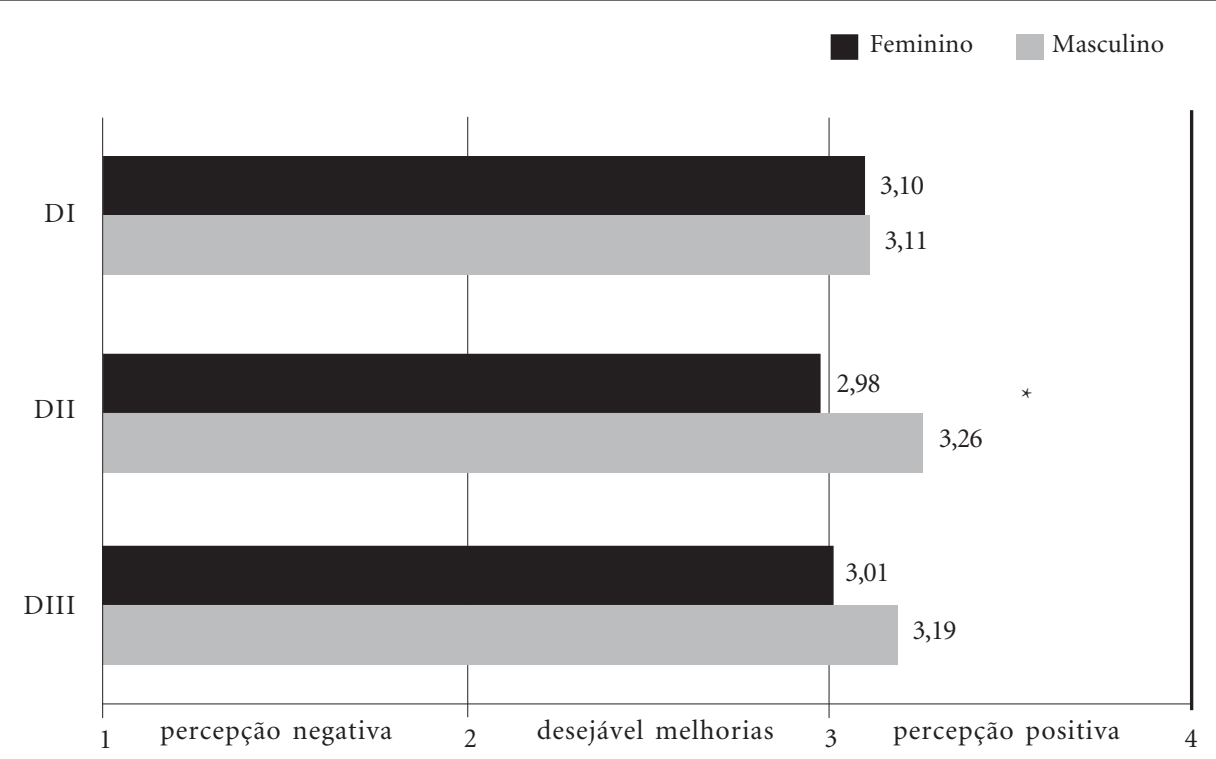

${ }^{*} \mathrm{p}<0,01$

D1: Dimensão I - Guia Alimentar Digital como ferramenta intuitiva e auto-explicativa

D2: Dimensão II - Guia Alimentar Digital como promotor de escolhas alimentares saudáveis

D3: Dimensão III - Guia Alimentar Digital como promotor da transição para o peso adequado

Figura 1. Média do perfil atitudinal entre os usuários do Guia Alimentar Digital. Brasil, 2012.

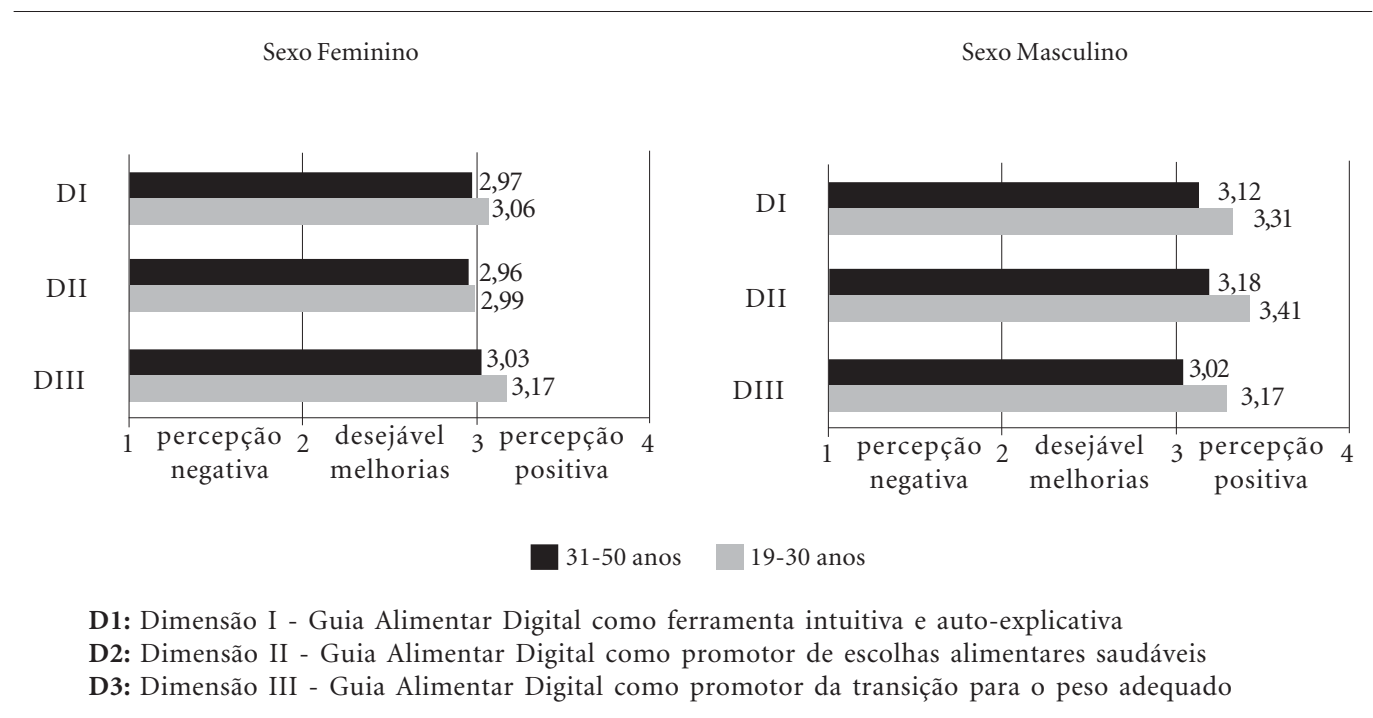

Figura 2. Média do perfil atitudinal sobre a avaliação do Guia Alimentar Digital. Brasil, 2012.

sição para o peso ideal (Dimensão III); já a Dimensão II apresenta diferença significativa segundo o sexo, sendo mais positiva para os homens, demonstrando que as mulheres possuem uma percepção mais crítica. Com o intuito de alcan- çar uma percepção mais positiva entre as mulheres, é importante considerar que se espera haver compreensões diferentes entre os sexos; Grohmann e Battistella ${ }^{14}$ apontam que as mulheres são mais orientadas por aspectos interpessoais $e$ 
com comportamentos expressivos, enquanto que homens são mais orientados pelo desempenho. Na concepção do aplicativo, pretendeu-se oferecer uma ferramenta para promoção da consciência sobre escolhas alimentares apropriadas o que talvez faça mais sentido entre os usuários do sexo masculino.

Homens mais jovens, com 19 a 30 anos, demonstraram maior facilidade de uso do GAD, maior percepção sobre capacidade de construir uma alimentação saudável e mais facilidade na transição para o peso ideal comparativamente a homens com mais de 31 anos. Das três dimensões, a que se refere à promoção de escolhas alimentares saudáveis foi a que reuniu assertivas com maiores pontuações, resultando em um valor médio de 3,41 pontos entre homens com 19 a 30 anos (Figura 1).

Mulheres entre 19 e 30 anos demonstraram maior facilidade de uso do GAD e mais facilidade na transição para o peso ideal. Já em relação à capacidade de construir uma alimentação saudável, a diferença entre os estágios de vida não é expressiva. Das três dimensões, a que se refere à facilidade de uso do GAD foi a que reuniu assertivas com maiores pontuações, resultando em um valor médio de 3,17 pontos entre mulheres com 19 a 30 anos (Figura 1).

- Dimensão I - GAD como uma ferramenta intuitiva e autoexplicativa

Os usuários mostraram estar inclinados a concordar $(3,59)$ com a assertiva 9: A atualização do peso no aplicativo é fácil. A boa organização do aplicativo é reiterada na assertiva 6 que $\operatorname{diz} A$ definição do peso meta no aplicativo é fácil com 3,51 pontos, seguida das assertivas $24 \mathrm{O}$ aplicativo é fácil de usar com 3,35 pontos e da $20 E$ fácil compreender as informações que estão contidas na tela 'Acompanhamento' do aplicativo com 3,14 pontos.

Alguns comentários indicam a boa usabilidade do aplicativo:

Gosto do aplicativo e uso há anos; me ajuda muito. Mas acho que, como é uma ferramenta que existe há tempos e vem se sofisticando, poderia dar passos mais ousados [...] uma tela 'acompanhamento' que excluisse carne para os vegetarianos ou tivesse funções específicas para os intolerantes à lactose. F, 31 a 50 anos.

E ainda,

O aplicativo é excelente! Gostaria de encontrar nele duas situações: 1. Opção vegetariana [...]. 2. [...] uma tabela de marcas/restaurantes com os dados dos alimentos mais consumidos, 3. Contador de calorias de atividades físicas. F, 18 a 30 anos.
Os usuários do aplicativo o avaliam positivamente quanto ao seu uso, mas sugerem melhorias como indicam os comentários a seguir:

Considero o aplicativo o melhor existente no Brasil [...] e aprecio bastante a distribuição dos alimentos de acordo com o grupo alimentar. Algumas sugestões de melhorias: gráficos para acompanhar evolução do peso, [...] mecanismo de recorrência de alimentos ou refeições para diferentes dias [...]. M, 31 a 50 anos.

A assertiva 22 afirma Estou satisfeito com a distribuição das calorias entre as refeições fornecidas pelo aplicativo e obteve 2,86 pontos, os quais correspondem à percepção de concordância na maioria das vezes. Neste caso, acreditava-se que os usuários não fossem concordar com a distribuição do valor energético ofertado entre as seis refeições contidas no aplicativo; entretanto, a maior parte dos usuários revelou satisfação com o que foi sugerido, embora quando se analisa a resposta segundo o sexo verifica-se que os homens de fato discordaram e as mulheres não. $\mathrm{O}$ mesmo aconteceu com a assertiva 18 que dizia Eu consigo alterar a faixa calórica proposta pelo aplicativo com facilidade; ao considerar o total de respostas, aqui também houve concordância na maioria das vezes com 2,70 pontos.

Eu costumo consumir mais calorias pela manhã e menos a noite do que o sugerido pelo aplicativo. Jantar pouco e cedo contribuiu muito para alcançar a meta de peso. M, 18 a 30 anos.

Já as assertivas relativas ao detalhamento do uso revelaram haver um pouco mais de dificuldade embora seja aproximada de uma boa percepção; isto indica a necessidade de refinamento de alguns aspectos operacionais do Guia. A assertiva 12 dizia:

A busca de alimentos no aplicativo é simples, pressupondo-se a concordância plena (4 pontos); os usuários tenderam a discordar na maioria das vezes com 2,86 pontos.

É difícil encontrar alguns alimentos, mesmo os mais simples e rotineiros. M, 18 a 30 anos.

Acredito que ainda é necessário incluir com maior frequência, alimentos na lista de opções, assim como pratos comuns na nossa culinária ou em restaurantes normalmente frequentados [...]. M, 18 a 30 anos.

A assertiva 15 tratava da inserção dos alimentos consumidos onde se afirmava Inserir os alimentos que consumi no aplicativo é uma tarefa simples e a população estudada discordou na maioria das vezes com 2,79 pontos.

- Dimensão II - GAD como promotor de escolhas alimentares saudáveis 
O propósito desta dimensão é resultado de uma construção que requer melhoria de hábitos e de estilo de vida; assim, trata do resultado do processo educativo para orientar a opção por alimentos saudáveis. O processo de educação alimentar e nutricional tem por objetivo a mudança de comportamento do indivíduo frente às escolhas alimentares ${ }^{4}$; compreender os determinantes do comportamento é o ponto mais importante para a capacitação do indivíduo, a fim de desenvolver e sustentar novas atitudes ${ }^{25,26}$.

A assertiva 19 afirmava Estou satisfeito com o número de refeições sugerido pelo aplicativo; acreditava-se que a discordância total seria prevalente e os usuários tenderam, de fato, a discordar na maioria das vezes, com 3,41 pontos. Os benefícios do fracionamento da energia ao longo do dia sobre indicadores de saúde são conhecidos há mais de quatro décadas ${ }^{27,28}$; embora a frequência de refeições por dia apresente tendência decrescente $^{29}$. Por outro lado, a assertiva 14 dizia $O$ aplicativo contribui para melhorar a qualidade da minha alimentação e a maior parte dos usuários concordou na maioria das vezes com 3,24 pontos. Aqui o estudo acreditava que esta percepção aconteceria e em função disso foram atribuídos 4 pontos à concordância plena.

Este bom resultado é referendado nas assertivas 11 (3,21 pontos) A tela 'Acompanhamento' do aplicativo contribui para percepção do consumo de cada grupo de alimentos, na 2 (3,19 pontos) $O$ aplicativo melhora a qualidade da minha alimentação e na 5 (3,08 pontos) A qualidade da minha alimentação melhorou com o uso do aplicativo porque aprendi a usar quantidades adequadas de alimentos, ou seja, vários elementos do aplicativo demonstraram muita assertividade no processo de construção de uma nova consciência alimentar dos usuários.

Este estudo apresenta, como limitação, a falta de informações socioeconômicas dos usuários; contudo, o aplicativo só estava disponível, à época da coleta de dados, na plataforma iOS. Esta plataforma operava no aparelho celular de mais elevado preço no mercado brasileiro para o período; assim, entende-se que o perfil socioeconômico dos respondentes não deve trazer viés para a interpretação dos dados.

Estudos sobre uso de tecnologias digitais têm mostrado o interesse de usuários sobre nutrição e saúde. Entre norte-americanos, o tema nutrição é frequente nas buscas de quase 50\% dos usuários, especialmente entre os adultos jovens ${ }^{19,30}$. O desenvolvimento de um aplicativo para Guia Alimentar com potencial de contri- buir para a melhor percepção sobre porcionamento e variedade da dieta pode promover melhora no padrão alimentar ${ }^{17,30,31}$.

. Dimensão III - GAD como promotor da transição para o peso adequado

As assertivas 4 (3,39 pontos) O aplicativo me ajuda a perceber as quantidades de alimentos que consumo, fazendo com que eu altere o tamanho das porções; 10 e 21, ambas com 3,26 pontos Poder alterar a faixa calórica proposta pelo aplicativo contribui para a redução de peso e $O$ uso do aplicativo contribui para o alcance da meta de peso corroboraram na afirmação sobre a assertividade da Dimensão II. Estes resultados sugerem que a construção de uma nova consciência alimentar pode ser contributiva para a adequação de peso, quando necessário.

Entretanto, as assertivas 16 (2,96 pontos) e 13 (2,73 pontos) apontam para alguns ajustes uma vez que enunciam Consulto a tela 'Acompanhamento' do aplicativo com frequência para analisar a evolução do consumo das calorias e $O$ aplicativo não fornece ferramentas suficientes para o alcance da minha meta de peso. Na assertiva 16 os usuários discordaram denotando alguma dificuldade na consulta e na 13 concordam que o aplicativo é insuficiente para o alcance do peso desejado.

A principal limitação deste estudo refere-se à amostra de conveniência de 80 respondentes; contudo, considerando os critérios de inclusão e os resultados do estudo de validação das asserções, os achados sobre a percepção dos usuários são consistentes e comparáveis aos obtidos por outros grupos que trabalharam com casuísticas semelhantes ${ }^{25,27,32}$.

\section{Conclusão}

O aplicativo Guia Alimentar Digital, segundo a percepção de seus usuários, apresenta boa usabilidade, a considerar as análises das dimensões referentes ao seu desempenho como ferramenta intuitiva e autoexplicativa, promotora de escolhas alimentares saudáveis e de transição para o peso adequado. A escuta ao usuário indica que os pontos fortes para a aceitação da ferramenta referem-se ao auxílio que representa para o correto porcionamento dos alimentos, bem como a composição do dia alimentar com opções dos diferentes grupos alimentares; por outro lado, o aplicativo deve ser aprimorado no que se refere à facilidade para inserção dos alimentos e acesso aos gráficos de acompanhamento, já disponíveis, mas ainda não percebidos pelo usuário. 


\section{Colaboradores}

S Caivano participou da concepção e delineamento do trabalho; coleta, análise e interpretação dos dados; redação e revisão crítica do artigo e aprovação da versão a ser publicada. BJ Ferreira participou da concepção e delineamento do trabalho; análise e interpretação dos dados; redação e revisão crítica do artigo e aprovação da versão a ser publicada. SMA Domene participou da concepção e delineamento do trabalho; análise dos dados; redação e revisão crítica do artigo e aprovação da versão a ser publicada.

\section{Referências}

1. Word Health Organization (WHO). Obesity and overweight. 2013. [Internet]. [acessado 2014 abr 2]. Disponível em: http://www.who.int/mediacentre/ factsheets/fs311/en/index.html\#

2. Instituto Brasileiro de Geografia e Estatística (IBGE). Antropometria e estado nutricional de crianças, adolescentes e adultos no Brasil. Brasil. [Internet]. [acessado 2013 jul 15]. Disponível em: http://www.ibge. gov.br/home/presidendia/noticias/noticia_ visualiza.php?id_noticia $=1699$ \&id_pagina $=1$

3. Noronha J, Hysen E, Zhang H. PlateMate: crowdsourcing nutrition analysis from food photographs. In: UIST'11 Proceedings og the 24th annual ACM symposium on user interface software and technology. Washington: Harvard School of Engineering and Applied Sciences; 2011. p. 1-12

4. Teixeira PDS, Reis BZ, Vieira DAS, Costa D, Costa JO, Raposo OFF, Wartha ERSA, Netto RSM. Intervenção nutricional educative como ferramenta eficaz para mudanças de hábitos alimentares e peso corporal entre praticantes de atividade física. Cien Saude Colet 2013; 18(2):347-356.

5. Liu C, Zhu Q, Holroyd KA, Seng EK. Status and trends of mobile-health applications for iOS devices: a developer's perspective. J Syst Software 2011; 84(11):2022-2033.

6. Parker AG, Harper R, Grinter RE. Celebratory health technology. J Diab Science Techny 2011; 5(2):319324.

7. Penn L, Boeing H, Boushey CJ, Dragsted LO, Kaput J, Scalbert A, Welch AA, Mathers JC. Assessment of dietary intake: NuGO symposium report. Genes Nutr 2010; 5(3):205-213.

8. Thompson FE, Subar AF, Loria CM, Reedy JL, Baranowshi $\mathrm{T}$. Need for technological innovation in dietary assessment. J Am Diet Assoc 2011; 110(1):48-51.

9. Instituto Brasileiro de Geografia e Estatística (IBGE). Brasil, redes: linhas telefônicas e assinantes de telefonia celular. Brasil. 2010. [Internet]. [acessado 2013 jul 15]. Disponível em: http://www.ibge.gov.br/oaisesat/main.php 
10. Instituto Brasileiro de Geografia e Estatística (IBGE). Sala de imprensa: acesso à internet e posse de telefone móvel celular para uso pessoal. Brasil. 2009. [acessado 2013 jul 13]. Disponível em: http://www. ibge.gov.br/home/noticias/notica_visualiza.php? id_noticia $=1517$

11. Instituto Brasileiro de Geografia e Estatística (IBGE). Sala de imprensa o setor de tecnologia da informação e comunicação no Brasil. Brasil. 2009. [Internet]. [acessado 2013 jul 13]. Disponível em: http://www. ibge.gov.br/home/presidencia/noticias/noticias_ visualiza.php?id_noticia $=1344 \&$ id $\_$pagina $=1 \&$ titulo=IBGE-divulga-estudo-inedito-sobre-setorde-Tecnologia-da-Informacao-e-Comunicacao-nopais

12. Kenney M, Pon B. Structuring the smartphone industry: is the mobile internet OS platform the key? J Ind Compet Trade 2011; 11(3):239-261.

13. Moura AM. Apropriação do telemóvel como ferramenta de mediação em mobile learning - estudos de caso em contexto educativo [dissertação]. Braga: Universidade do Minho; 2010.

14. Grohmann ZM, Battistella LF. Homens e mulheres "aceitam" de maneira diferente? impacto do gênero no modelo (expandido) de aceitação da tecnologia - TAM. Inf \& Soc 2011; 21(1):175-189.

15. Likert R, Roslow S, Murphy G. A simple and reliable method of scoring the thurstone attitude scales. Pers Psychol 1993; 46(3):689-690.

16. Wakita T, Ueshima N, Noguchi H. Psychological distance between categories in the Likert Scale: comparing different numbers of options. EPM 2012; 72(4):533-546.

17. Willett WC, Skerrett PJ. Eat, drink, and be healthy: the Harvard Medical School Guide to Healthy Eating. Washington: Harvard School of Medicine Free Press; 2002.

18. Harvard School of Public Health. Guide to eating a healthy meal based on latest science. HSPH News. 2011. [Internet]. [acessado 2014 abr 2]. Disponível em: http://www.hsph.harvard.edu/news/press-releases/healthy-eating-plate/

19. Brandalise LT. Modelos de medição de percepção e comportamento [dissertação]. Cascavel: Universidade Estadual do Oeste do Paraná; 2006.

20. Nata RN. Progress in Education. In: Moraes SG, Justino ML, Jansen BF, Barbosa EP, Bruno, LFC, Pereira LAV. Development and validation of a strategy to assess teaching methods in undergraduate disciplines. Nova York: Nova Science Publishers Inc; 2012. p. 81-107.

21. Schimidt MJ. Understanding and using statistics basic concepts. Massachusetts: Health and Company; 1975.

22. Instituto Brasileiro de Geografia e Estatística (IBGE). Censo demográfico 2010. Brasil. 2010. [Internet]. [acessado 2014 abr 2]. Disponível em: http://www. censo2010.ibge.gov.br/apps/mapa/
23. Boushey C, Wright J, KD L, Ebert D, EJ D. Use of technology in children's dietary assessment. Eur J Clin Nutr 2009; 63(1):S50-S57.

24. Sevick MA, Zickmund S, Korytkowski M, Piraino B, Sereika S, Mihalko S, Snetselaar L, Stumbo P, Hausmann L, Ren D, Marsh R, Sakraida T, Gibson J, Safaien M, Starrett TJ, Burke LE. Design, feasibility, and acceptability of an intervention using personal digital assistant-based self-monitoring in managing type 2 diabetes. Contemp Clin Trials 2008; 29(3):396409.

25. Toral N, Slater B. Abordagem do modelo transteórico no comportamento alimentar. Cien Saude Colet 2007; 12(6):1641-1650.

26. Fonseca AB, Souza TSN, Frozi DS, Pereira RA. Modernidade alimentar e consumo de alimentos: contribuições sócio-antropológicas para a pesquisa e nutrição. Cien Saude Colet 2011; 16(9):3853-3862.

27. Fabry P, Hejl Z, Fodor J, Braun T, K. Z. The frequency of meals: its relation to overweight, hypercholesterolaemia, and decreased glucose tolerance. Lancet 1964; 284(7360):614-615.

28. La Bounty PM, Campbell BI, Wilson J, Galvan E, Berardi J, Kleiner SM, Kreider RB, Stout JR, Ziegenfuss, Tim, Spano M, Smith A, Antonio J. International society of sports nutrition position stand: meal frequency. J Int Soc Sports Nutri 2011; 8(4):1-12.

29. Neumark-sztainer D, Wall M, Fulkerson JA, Larson $\mathrm{N}$. Changes in the frequency of family meals from 1999 to 2010 in the homes of adolescents: trends by sociodemographic characteristics. J Adol Health 2013; 52(2):201-206.

30. Chisolm DJ. Does online health information seeking act like a health behavior?: a test of the behavioral model. Telemed E-Health 2010; 16(2):154-160.

31. Rowe S, Alexander N, Almeida N, Black R, Burns R, Bush L, Crawford P, Keim N, Kris-etherton P, Weaver C, Guidelines D, Guidelines TD, States U, Guidelines D, Committee A, Americans A, Council FI, Control D, Horn LV. Food Science Challenge: translating the dietary guidelines for americans to bring about real behavior change. JFS 2011; 76(1):29-37.

32. Rusin M, Arsand E, Hartvigsen G. Functionalities and input methods for recording food intake: a systematic review. Int J Med Inform 2013; 82(8):653-664.

Artigo apresentado em 20/07/2013

Aprovado em 27/08/2013

Versão final apresentada em 09/09/2013 